\title{
Onset of Fast Reconnection in Hall Magnetohydrodynamics Mediated by the Plasmoid Instability
}

\author{
Yi-Min Huang:* A. Bhattacharjee, and Brian P. Sullivan \\ Center for Integrated Computation and Analysis of Reconnection and Turbulence and \\ Center for Magnetic Self-Organization in Laboratory and Astrophysical Plasmas, \\ University of New Hampshire, Durham, NH 03824
}

\begin{abstract}
The role of a super-Alfvénic plasmoid instability in the onset of fast reconnection is studied by means of the largest Hall magnetohydrodynamics simulations to date, with system sizes up to $10^{4}$ ion skin depths $\left(d_{i}\right)$. It is demonstrated that the plasmoid instability can facilitate the onset of rapid Hall reconnection, in a regime where the onset would otherwise be inaccessible because the SweetParker width is significantly above $d_{i}$. However, the topology of Hall reconnection is not inevitably a single stable X-point. There exists an intermediate regime where the single X-point topology itself exhibits instability, causing the system to alternate between a single X-point geometry and an extended current sheet with multiple X-points produced by the plasmoid instability. Through a series of simulations with various system sizes relative to $d_{i}$, it is shown that system size affects the accessibility of the intermediate regime. The larger the system size is, the easier it is to realize the intermediate regime. Although our Hall MHD model lacks many important physical effects included in fully kinetic models, the fact that a single X-point geometry is not inevitable raises the interesting possibility for the first time that Hall MHD simulations may have the potential to realize reconnection with geometrical features similar to those seen in fully kinetic simulations, namely, extended current sheets and plasmoid formation.
\end{abstract}

*Electronic address: yimin.huang@unh.edu 


\section{INTRODUCTION}

Magnetic reconnection is thought to be the underlying mechanism that powers explosive events such as flares, substorms, and sawtooth crashes in fusion plasmas[1]. Such events commonly feature impulsive onset, whereby the system exhibits a sudden increase in the reconnection rate [2]. In classic Sweet-Parker theory [3, 4], based on resistive magnetohydrodynamics (MHD), the reconnection site has the structure of a thin current sheet of length $L$, which is of the order of the system size, and a width $\delta_{S P} \simeq L / \sqrt{S}$, where the Lundquist number $S$ is related to the length $L$, the Alfvén speed $V_{A}$, and the resistivity $\eta$ by the relation $S \equiv L V_{A} / \eta$. The plasma outflow speed from the reconnection site is approximately $V_{A}$, and the inflow speed, which is a measure of the reconnection rate, is approximately $V_{A} / S^{1 / 2}$ under quasi-steady conditions. In most plasmas of interest, the Lundquist numbers are very high. Consequently, the Sweet-Parker reconnection rates are usually several orders of magnitude too slow to account for the observed rate of energy release after onset. The strong dependence of the reconnection rate on $S$ in the Sweet-Parker theory has led to a broad consensus that the solution to the onset problem for high- $S$ plasmas lies outside the domain of resistive MHD, and requires the inclusion of collisionless effects. In particular, for two-dimensional (2D) configurations without a guide field, a precise criterion has been proposed that accounts for a slow growth phase (identified as a Sweet-Parker phase in many cases of interest), followed by rapid onset caused by the Hall current, which is a signature of the decoupling of electron and ion motion at scales below the ion skin depth $d_{i}[5-8]$. (Here $d_{i}=c / \omega_{p i}$, where $c$ is the speed of light and $\omega_{p i}$ is the ion plasma frequency.) The criterion predicts that when $\delta_{S P}<d_{i}$, the system will spontaneously make a transition to a rapid reconnection phase, with an inflow velocity $\sim 0.1 V_{A}$. This criterion has been tested extensively by numerical simulations [5] 8] as well as controlled laboratory experiments [9].

The recent discovery of a linear, super-Alfvénic plasmoid instability [10] in high- $S$ plasmas raises qualitatively new questions for the criterion stated above. It has long been known that the Sweet-Parker reconnection layer can become unstable to a secondary tearing instability. However, only recently has a precise linear study shown that the linear growth rate $\gamma$ of the instability scales as $\gamma \sim S^{1 / 4}\left(V_{A} / L\right)$. The positive exponent of $S$ yields high growth rates for high $S$ plasmas, whereas most resistive instabilities scale with $S$ to some negative fractional power. This seemingly counterintuitive result can actually be deduced from the dispersion 
relation for classical tearing modes[11] with one crucial new insight: the Sweet-Parker layer supports an increasingly singular current sheet as $S \rightarrow \infty$.[12] Furthermore, even within the framework of resistive MHD, this linear instability leads to a nonlinear regime where the reconnection rate becomes nearly independent of $S$, with an inflow velocity $\sim 10^{-2} V_{A} \cdot[12,13$. The original Sweet-Parker current sheet breaks up into a chain of plasmoids and a sequence of shorter but thinner current sheets, with widths much smaller than $\delta_{S P} .[12,14,15]$

The presence of the plasmoid instability in high- $S$ systems uncovers a deep flaw in the Sweet-Parker model, and raises questions about the conventional scenario of the onset of Hall reconnection. Because secondary current sheets are thinner than the primary Sweet-Parker current sheet, potentially they may trigger onset of Hall MHD (or kinetic) reconnection when the widths reach the $d_{i}$ scale, even in systems where the original onset criterion $\delta_{S P}<d_{i}$ is not met. Shibata and Tanuma [16] proposed just this scenario in an insightful paper, years before the recent spate of interest in this topic. Recently, numerical studies have been carried out with fully kinetic particle-in-cell (PIC) simulations including a collision operator by Daughton et al. [14] and Hall MHD simulations by Shepherd and Cassak [17] confirming the role of the plamoid instability in triggering onset of Hall (or kinetic) reconnection. However, the PIC and Hall MHD simulations, discussed in Refs. [14] and [17], show qualitatively very different behaviors after onset. Whereas PIC simulations continue to exhibit copious generation of plasmoids, the Hall MHD solutions appear to settle down to a single stable X-point state with all plasmoids expelled. This apparent qualitative difference between the two types of simulations raises the following important questions of principle: Does the onset of Hall reconnection in Hall MHD models inevitably lead to a Hall current dominated regime in which all plasmoids are expelled? Can Hall MHD realize current sheet geometries qualitatively similar to those seen in fully kinetic simulations, where new plasmoids are constantly generated?

In this paper, we address these questions by means of the largest two-dimensional resistive Hall MHD reconnection simulations ever carried out, with the ratio $L / d_{i}$ ranging from $2.5 \times 10^{3}$ to $1.0 \times 10^{4}$, in a configuration of two coalescing magnetic islands. We confirm the previous results that the plasmoid instability can trigger the onset of Hall MHD reconnection in systems that do not meet the criterion $\delta_{S P}<d_{i}$ for onset. In addition, we demonstrate that the topology of Hall MHD reconnection is not inevitably a single stable X-point. There exists an intermediate regime where the single X-point topology itself exhibits instability, 
causing the system to alternate between a single X-point and an extended current sheet with multiple X-points produced by the plasmoid instability. Furthermore, through a series of simulations with various system sizes relative to $d_{i}$, we show numerical evidence supporting the idea that system size affects the accessibility of the intermediate regime. The larger the system size is, the easier it is to realize the intermediate regime.

The present study employs a simple Hall MHD model, which clearly lacks many important physical effects that are included in fully kinetic models. A constant resistivity instead of the Spitzer resistivity[18] is employed; an isothermal equation of state is employed and Ohmic heating is neglected; plasma pressure is assumed to be a scalar rather than a tensor; and electrons are assumed to be massless. Furthermore, it has been argued that in many collisionless or weakly collisional systems of interest the reconnection electric field typically

exceeds the Dreicer runaway field, [19] therefore classical resistivity cannot play a significant role. While these limitations merit further investigations, the fact that a single X-point geometry is not inevitable in Hall MHD simulations raises the interesting possibility for the first time that Hall MHD simulations may have the potential to realize reconnection with geometrical features similar to those seen in fully kinetic simulations, namely, extended current sheets and plasmoid formation.

\section{NUMERICAL MODEL}

Our simulations are based on resistive Hall MHD equations. These equations in normalized form are:

$$
\begin{gathered}
\partial_{t} \rho+\nabla \cdot(\rho \mathbf{u})=0 \\
\partial_{t}(\rho \mathbf{u})+\nabla \cdot(\rho \mathbf{u u})=-\nabla p+\mathbf{J} \times \mathbf{B}+\epsilon \mathbf{f}(\mathbf{x}, t) \\
\partial_{t} \mathbf{B}=-\nabla \times \mathbf{E} \\
\mathbf{u}_{e}=\mathbf{u}-d_{i} \frac{\mathbf{J}}{\rho} \\
\mathbf{E}=-\mathbf{u}_{e} \times \mathbf{B}-d_{i} \frac{\nabla p_{e}}{\rho}+\eta \mathbf{J}
\end{gathered}
$$

where $\rho$ is the plasma density, $\mathbf{u}$ is the ion velocity, $\mathbf{u}_{e}$ is the electron velocity, $p$ is the total pressure, $p_{e}$ is the electron pressure, $\mathbf{B}$ is the magnetic field, $\mathbf{E}$ is the electric field, $\mathbf{J}=\nabla \times \mathbf{B}$ is the electric current density, $\eta$ is the resistivity, and $d_{i}$ is the ion skin depth. 
Isothermal equations of state are assumed, i.e. $p_{e}=p_{i}=\rho T$, where $p_{i}$ is ion pressure, and $T$ is a constant temperature. The total pressure is $p=p_{e}+p_{i}=2 \rho T$. Electron inertia terms are neglected in the generalized Ohm's law, Eq. (5). The electron pressure term $-d_{i} \nabla p_{e} / \rho$ has been omitted in this study, because it does not contribute to the dynamics after taking the curl of $\mathbf{E}$ in Eq. (3), due to the isothermal equation of state. A weak random forcing term $\epsilon \mathbf{f}$ is added to the ion momentum equation, as was done in a previous study. [13] The normalizations of Eqs. (1) - (5) are based on constant reference values of the density $n_{0}$, and the magnetic field $B_{0}$. Lengths are normalized to the system size $L$, and time is normalized to the global Alfvén time $t_{A}=L / V_{A}$, where $V_{A}=B_{0} / \sqrt{4 \pi n_{0} m_{i}}$ and $m_{i}$ is the ion mass. The normalizations of physical variables are given by (normalized $\rightarrow$ physical units): $\rho \rightarrow \rho / n_{0} m_{i}, \mathbf{B} \rightarrow \mathbf{B} / B_{0}, \mathbf{E} \rightarrow c \mathbf{E} / B_{0} V_{A}, \mathbf{u} \rightarrow \mathbf{u} / V_{A}, p \rightarrow p / n_{0} m_{i} V_{A}^{2}, \mathbf{J} \rightarrow \mathbf{J} /\left(B_{0} c / 4 \pi L\right)$, and $d_{i} \rightarrow d_{i} / L \equiv \sqrt{m_{i} c^{2} / 4 \pi n_{0} e^{2}} / L$. In $2 \mathrm{D}$ simulations, the magnetic field is expressed in terms of the flux function $\psi$ and the out-of-plane component $B_{y}$ as $\mathbf{B}=\nabla \psi \times \hat{\mathbf{y}}+B_{y} \hat{\mathbf{y}}$. The variables $\psi$ and $B_{y}$ are stepped in the code. The governing equations are numerically solved with a massively parallel code HMHD, which is a two-fluid extension of the resistive MHD code used in previous studies.[12, 13] The numerical algorithm [20] approximates spatial derivatives by finite differences with a five-point stencil in each direction. The time-stepping scheme can be chosen from several options including a second-order accurate trapezoidal leapfrog method and various strong stability preserving Runge-Kutta methods. [21, 22] We employ the second-order accurate trapezoidal leapfrog method in this study. HMHD has the capability of nonuniform meshes that allows better resolution of the reconnection layer.

We employ the same simulation setup of two coalescing magnetic islands as in a previous study[13]. The $2 \mathrm{D}$ simulation box is the domain $(x, z) \in[-1 / 2,1 / 2] \times[-1 / 2,1 / 2]$. In normalized units, the initial magnetic field is given by $\mathbf{B}_{0}=\nabla \psi_{0} \times \hat{\mathbf{y}}$, where $\psi_{0}=$ $\tanh (z / h) \cos (\pi x) \sin (2 \pi z) / 2 \pi$. The parameter $h$, which is set to 0.01 for all simulations, determines the initial current layer width. The initial plasma density $\rho$ is approximately 1 , and the plasma temperature $T$ is 3 . The density profile has a weak nonuniformity such that the initial condition is approximately force-balanced. The initial peak magnetic field and Alfvén speed are both approximately unity. The plasma beta $\beta \equiv p / B^{2}=2 \rho T / B^{2}$ is greater than 6 everywhere. Perfectly conducting and free slipping boundary conditions are imposed along both $x$ and $z$ directions. Specifically, we have $\psi=0, \mathbf{u} \cdot \hat{\mathbf{n}}=0, \hat{\mathbf{n}} \cdot \nabla(\hat{\mathbf{n}} \times \mathbf{u})=0$, $\hat{\mathbf{n}} \cdot \nabla \rho=0$, and $B_{y}=0$ on the boundaries (here $\hat{\mathbf{n}}$ is the unit normal vector to the bound- 


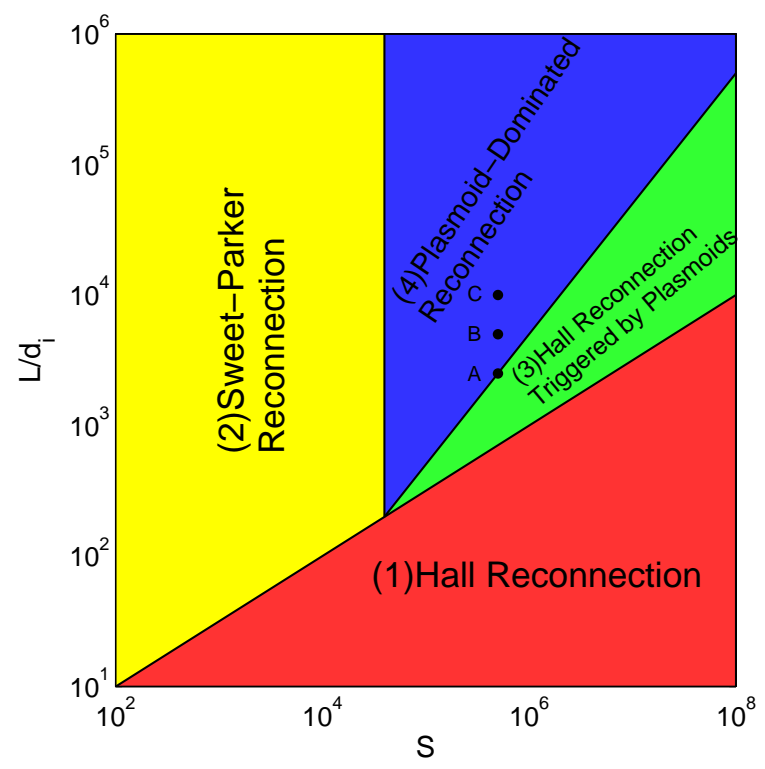

Figure 1: (Color online) The parameter space in the phase diagram is divided into four regions. (1) Hall reconnection: $d_{i}>\delta_{S P}$. (2) Sweet-Parker reconnection: $d_{i}<\delta_{S P}$ and $S<S_{c}$.

Hall reconnection triggered by plasmoids: $\delta_{S P}\left(S_{c} / S\right)^{1 / 2}<d_{i}<\delta_{S P}$. (4) Plasmoid-dominated reconnection: $S>S_{c}$ and $\delta_{S P}\left(S_{c} / S\right)^{1 / 2}>d_{i}$. The dots denote the parameters for three different runs. All three runs have $S=5 \times 10^{5}$. The parameter $L / d_{i}$ is $2.5 \times 10^{3}$ for Run A, $5 \times 10^{3}$ for Run $\mathrm{B}$, and $10^{4}$ for Run C, respectively. A fourth run, Run D, from a previous resistive MHD study [13], corresponds to $L / d_{i} \rightarrow \infty$, therefore is not shown.

ary). Only the upper half of the domain $(z \geq 0)$ is simulated, and solutions in the lower half are inferred by symmetries. The computational mesh consists of $6400 \times 1024$ grid points. The grid points along $z$ are strongly concentrated around $z=0$, with the smallest grid size $\Delta z=1.4 \times 10^{-5}$. The grid points along $x$ are weakly nonuniform, with the smallest grid size $\Delta x=1.2 \times 10^{-4}$ at $x=0$. For this system, the critical Lundquist number $S_{c}$ for onset of the plasmoid instability is approximately $4 \times 10^{4}$ in resistive $\operatorname{MHD}\left(d_{i}=0\right)$. [13]

\section{A PHASE DIAGRAM}

It is useful to map the numerical solutions discussed below into a phase diagram, shown in Figure 1. When the Hall effect is included, the system is characterized by two important dimensionless parameters: $S$ and $L / d_{i}$. The parameter space of $S$ and $L / d_{i}$ may be divided qualitatively into four regions. The Hall reconnection regime is realized when the conven- 
tional criterion $d_{i}>\delta_{S P}$ for onset of Hall reconnection is satisfied. Under this condition, we recover the standard results for the onset of Hall reconnection. [5-8] The Sweet-Parker reconnection regime is realized when neither the criterion for onset of Hall reconnection $d_{i}>\delta_{S P}$ nor that for the plasmoid instability $S>S_{c}$ are satisfied. In this regime, a stable, elongated Sweet-Parker current layer is formed. When the Lundquist number $S$ exceeds the critical value $S_{c}$ for onset of the plasmoid instability, two new possibilities emerge. If the secondary current sheets cascade down to widths at the $d_{i}$ scale, we may expect onset of Hall reconnection. On the other hand, if the secondary current sheets never reach the $d_{i}$ scale, the reconnection may proceed in a manner similar to that in resistive MHD. To delineate the border between these two regimes, an estimate for the widths of secondary current sheets is needed. In a previous resistive MHD study, we found that a good estimate for the average width of the secondary current sheets is obtained by requiring that they obey Sweet-Parker scaling, with a length that keeps them marginally stable. That gives an average width $\delta \sim \delta_{S P}\left(S_{c} / S\right)^{1 / 2} \sim L S_{c}^{1 / 2} / S$.[13] We denote the regime where $\delta<d_{i}$ as "Hall reconnection triggered by plasmoids", and the regime where $\delta>d_{i}$ as "plasmoid-dominated reconnection" to characterize their different possible behaviors. Note that statistical deviations from the average width can and do occur. 13] As individual secondary current sheets can be significantly thinner than the average width, we expect the "Hall reconnection triggered by plasmoids" region to be larger than depicted in Figure 1. We caution that since high- $S$, large-scale Hall MHD reconnection is largely unexplored, Figure 1 cannot be regarded as a complete picture because it includes ranges of parameter space where no simulations exist. Even the critical Lundquist number $S_{c}$ and the secondary current sheet width $\delta$ could be modified by the presence of the Hall effect. Furthermore, the criterion for onset $\delta<d_{i}$ is only accurate up to a numerical factor of order unity. For these reasons, the delineation of different regimes in Figure 1 may not be very precise. Nonetheless, Figure 1 serves well in guiding the choice of simulation parameters where interesting physics may arise.

The primary interest of this work is to explore the two new regimes where the plasmoid instability may play an important role. This study includes three new runs (Run A to C), with corresponding parameters marked on Figure 1. A fourth run, Run D, from a previous resistive MHD study[13], is included for comparison. We fix $S=5 \times 10^{5}$ for all runs. The parameter $L / d_{i}$ is $2.5 \times 10^{3}$ for Run A, $5 \times 10^{3}$ for Run B, and $10^{4}$ for Run $\mathrm{C}$, respectively. We have chosen parameters for the new runs such that after the onset of 


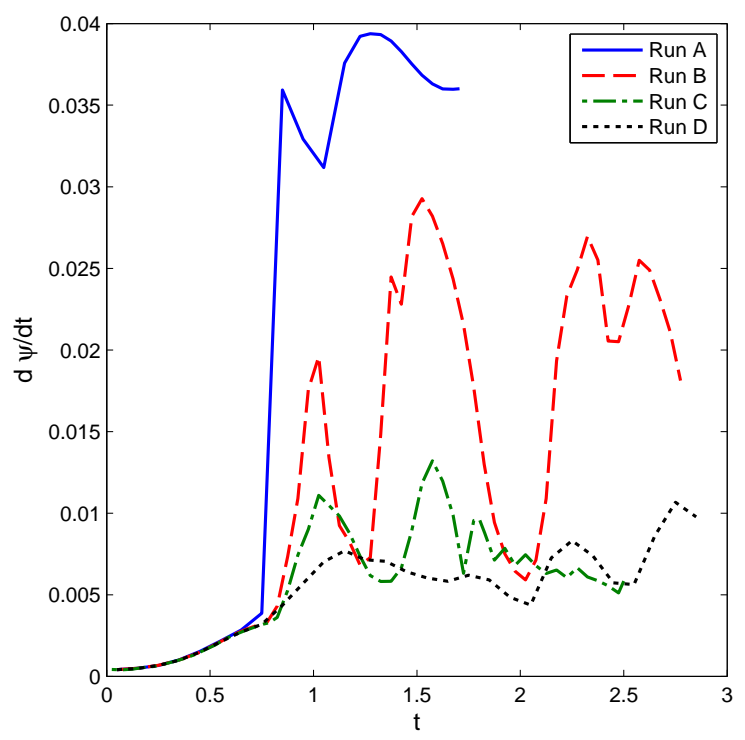

Figure 2: (Color online) The reconnection rate as a function of time, for four different runs.

the plasmoid instability the current sheets would have widths (estimated from the scaling law $\delta \sim \delta_{S P}\left(S_{c} / S\right)^{1 / 2}$ ) ranging from $d_{i}$ (Run A) to $4 d_{i}$ (Run $\mathrm{C}$ ). This is the parameter regime where we may expect to observe a transition from the "Hall reconnection triggered by plasmoids" regime to the "plasmoid-dominated reconnection" regime, depending on the ratio $\delta / d_{i}$. The initial condition and governing parameters for these runs allow a clear separation of length scales: the drivers of reconnection (the two merging islands) are on the largest scale $\sim 1$; the initial current layer width $\sim 0.01$; the Sweet-Parker width $\sim 10^{-3}$; and the ion skin depth $d_{i} \sim 1-4 \times 10^{-4}$. Therefore, the simulations cover all distinct stages of reconnection from the initial current sheet thinning to the onset of plasmoid instability, which subsequently may or may not lead to onset of Hall reconnection.

\section{SIMULATION RESULTS}

Our primary diagnostics are the reconnection rate and the length and width of the main reconnection current sheet. The reconnection rate is measured as the time derivative of the reconnected magnetic flux. In the presence of the plasmoid instability, the reconnection layer generally contains multiple current sheets at a given time. We define the main reconnection current sheet as the one located where the two primary coalescing islands touch each other. This is the (generally unique) point where the separatrix flux surface bounding the two 


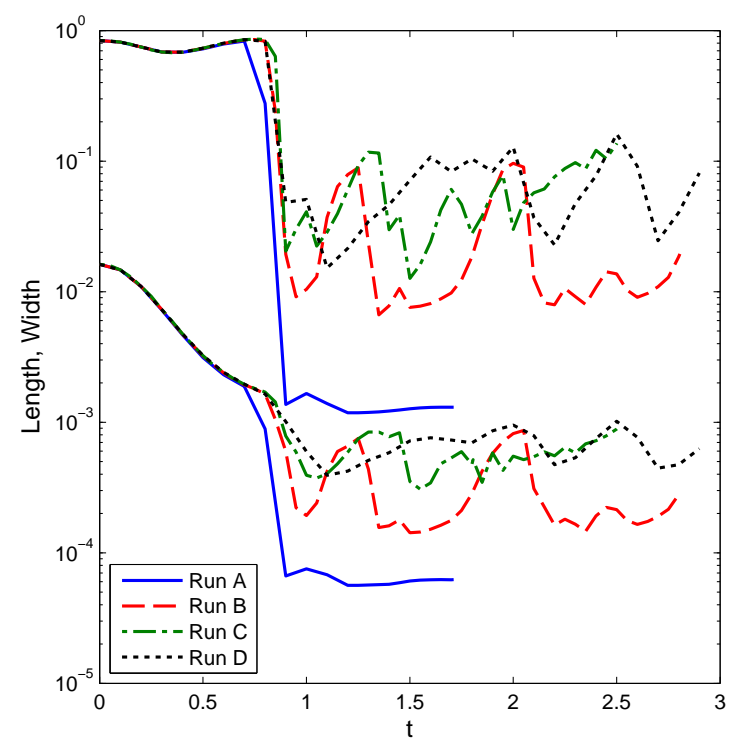

Figure 3: (Color online) The length (upper curve) and width (lower curve) of the main reconnection current sheet as a function of time, for four different runs.

merging islands intersects itself. For example, in the second panel of Figure 5, the main reconnection current sheet is the one at the center, between $x=0$ and $x=0.1$. The length and width are measured as the full width at quarter maximum.

Figure 2 and 3 show, respectively, the time-histories of the reconnection rate, and the length and width of the main reconnection current sheet for four different runs. Initial current sheet thinning occurs from $t=0$ to $t=0.7$. During this period, the four runs are very similar because the Hall current has yet to play an important role. The current sheet width thins from the initial $\delta \sim 10^{-2}$ down to $\delta \sim \delta_{S P} \sim 10^{-3}$. Meanwhile, the reconnection rate gradually rises to $3 \times 10^{-3}$. The plasmoid instability sets in at approximately $t=0.7$. Thereafter, the three new runs exhibit qualitatively different behaviors. In Run A, the plasmoid instability immediately triggers a strong onset of Hall reconnection, which expels all the plasmoids, and the system is left with a single X-point. After that, the system reaches a quasi-steady state with the reconnection rate and current sheet geometry approximately time-independent. This run gives the highest reconnection rate (up to 0.04) of the four runs, and the current sheet is also the shortest and narrowest. The aspect ratio (width/length) of the current sheet in the quasi-steady state is approximately 1/20. Figure 4 shows the out-of-plane electric current density, overlaid with magnetic field lines, in the whole domain at $t=1.5$. Dashed lines denote the separatrices which are the field lines that separate the 


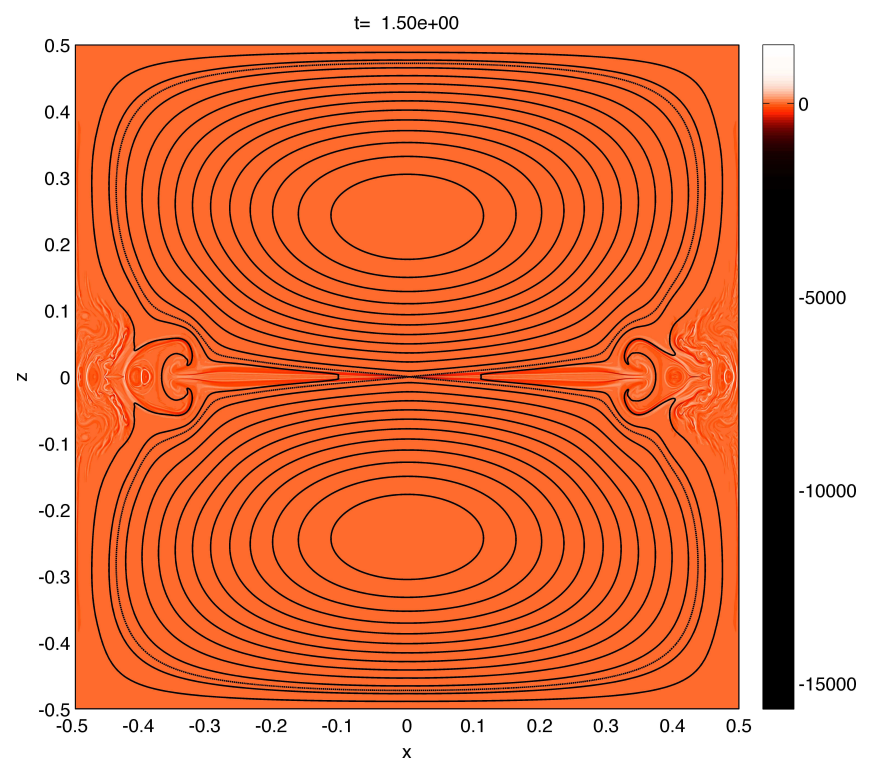

Figure 4: (Color online) Out-of-plane electric current density at $t=1.5$ for Run A, overlaid with magnetic field lines, in the whole simulation box. Dashed lines indicate separatrices, which are the field lines that separate the two merging islands.

two merging islands. The reconnection site clearly shows a Petschek-like geometry with the separatrices opening up in the downstream region.

In Run B (see Figure 5 for a few snapshots of the key stages), the plasmoid instability does not immediately lead to onset of Hall reconnection. An onset occurs at approximately $t=1.3$, triggered by a new plasmoid formed in the main reconnection current sheet. Subsequently all plasmoids are wiped out. However, it appears that Hall reconnection with a single X-point is unstable for this set of parameters, and the system makes a transition back to an extended current sheet. The current sheet length reaches a maximum $\left(\simeq 0.1=500 d_{i}\right)$ at $t=2$, whereupon it becomes unstable again and breaks up into plasmoids. This second onset of plasmoid instability leads to another onset of Hall reconnection, resulting in a single $\mathrm{X}$-point again. Conceivably, this cycle will continue repeatedly until the system runs out of flux. Indeed, towards the end of this run, we observe that the length and width of the main current sheet start to rise again (Figure 3). In this regime, which we have called the intermediate regime, the system is caught in between Hall reconnection with a single X-point, and plasmoid-dominated reconnection with multiple X-points. The resulting reconnection rate fluctuates strongly between 0.005 to 0.03 . For Run $\mathrm{C}$, because $d_{i}$ is well below the smallest scale caused by the plasmoid instability, the system never makes a transition to 


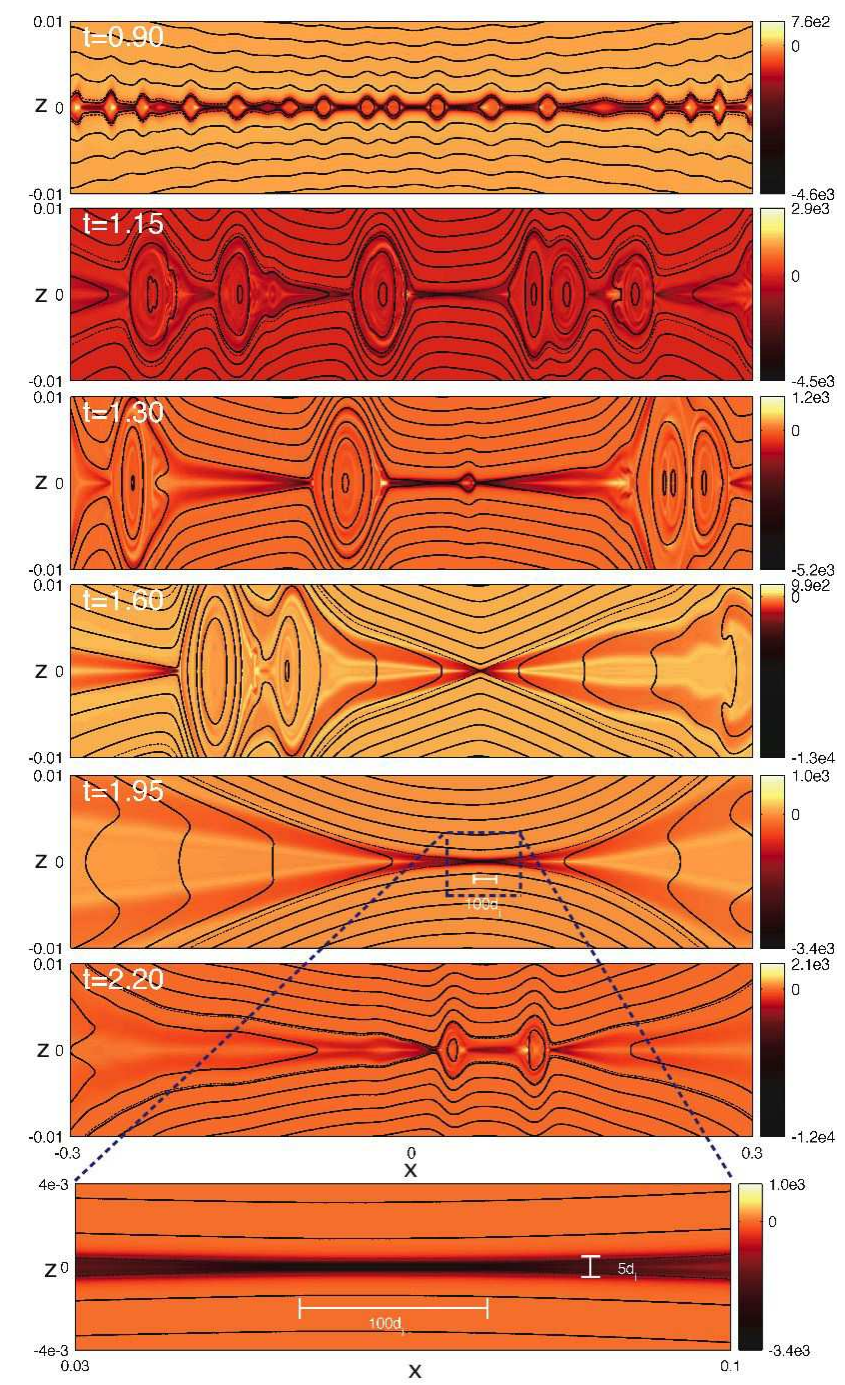

Figure 5: (Color online) Time sequence of the out-of-plane electric current density for Run B, overlaid with magnetic field lines. Dashed lines indicate separatrices. From top to bottom: (1) The Sweet-Parker current sheet breaks up into a chain of plasmoids. (2) The plasmoids grow in size; some of them are expelled to the downstream region; some of them coalesce to form larger plasmoids. (3) A new plasmoid forms at the main current sheet. (4) The formation of the new plasmoid leads to an onset of Hall reconnection that eventually expels all plasmoids. (5) The current sheet becomes extended again. (6) Subsequently, the extended current sheet breaks up into plasmoids, which lead to another onset of Hall reconnection. The bottom panel shows an expanded view of the extended current sheet at $t=1.95$ (enhanced online). 


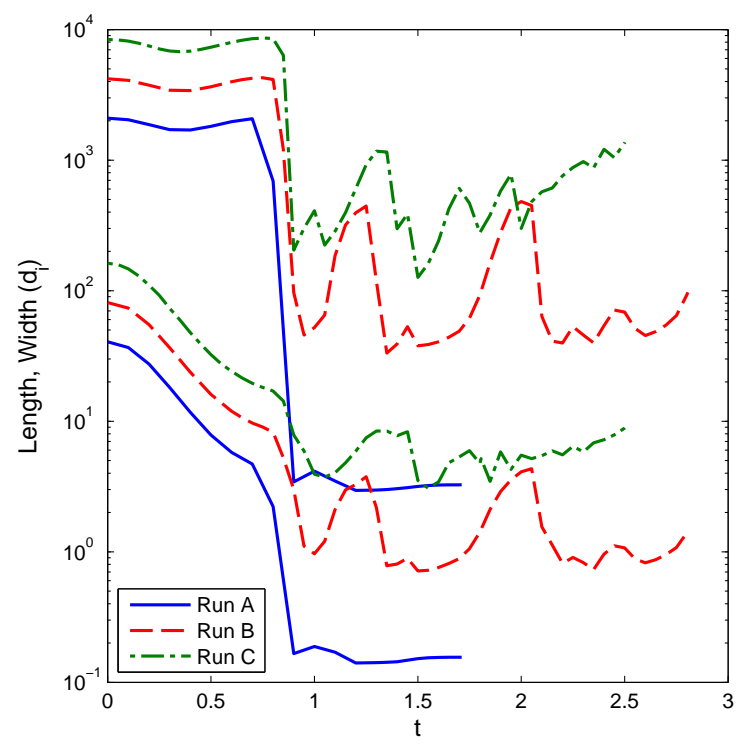

Figure 6: (Color online) The length (upper curve) and width (lower curve) of the main reconnection current sheet, normalized to the ion skin depth $d_{i}$, as a function of time for Run A to Run C.

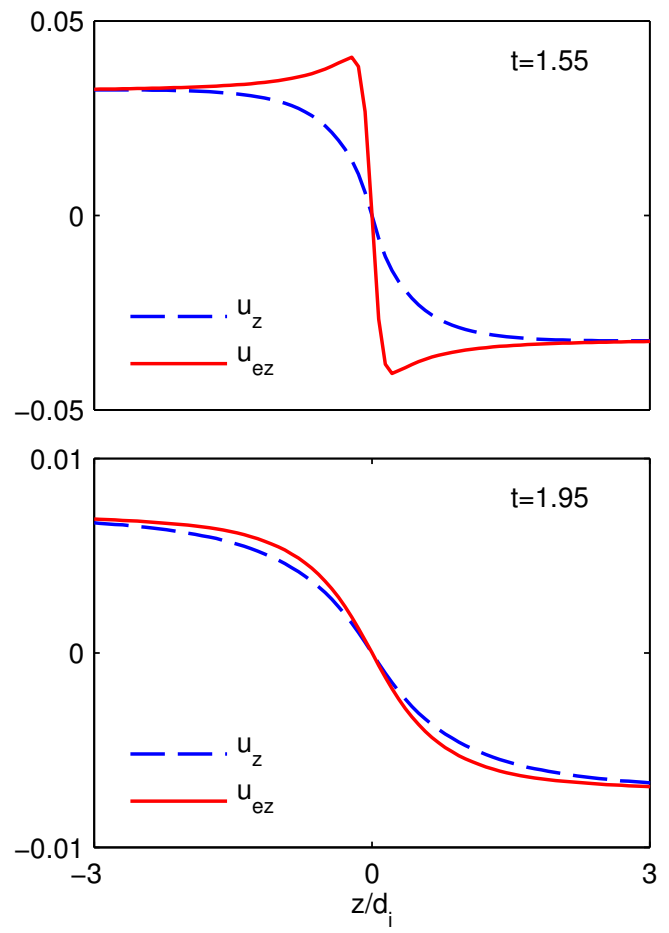

Figure 7: (Color online) The electron and ion flows along the inflow $(z)$ direction through the $\mathrm{X}$-point for Run B at $t=1.55$ and $t=1.95$. 
Hall reconnection. The reconnection rate from Run C, ranging from 0.005 to 0.013 , is similar to that from Run D, which is a resistive MHD simulation $\left(d_{i}=0\right)$.

In Figure 6 we replot the time histories of the length and width of the main current sheet as shown in Figure 3, but this time in units of $d_{i}$. Hall reconnection is characterized by the decoupling of ions and electrons at scales smaller than $d_{i}$, and the dissipation region where the frozen-in condition is broken is significantly smaller than the $d_{i}$ scale. Run A clearly exhibits these features, as the current sheet width during the quasi-steady phase is approximately $0.15 d_{i}$. On the other hand, the main current sheet in Run B is never significantly thinner than $d_{i}$. The minimum current sheet width is approximately $0.7 d_{i}$ in this run. (However, note that we measure the current sheet width by its full width at quarter maximum. Instead, if we measure by its half width at half maximum, as employed by Cassak et al. [8], the minimum width in Run B is $0.17 d_{i}$. This value is on par with the typical current sheet width of Hall solutions reported by Cassak et al.[8]) This suggests that the Hall reconnection after onset is not as robust as it is in Run A. Nonetheless, Run B clearly shows the characteristic of Hall reconnection, i.e. the decoupling of electron and ion flow at scales below $d_{i}$, when the current sheet width reaches the minimum. Figure 7 shows one-dimensional (1D) profiles of the electron and ion flows along the $z$ direction through the $\mathrm{X}$-point, at $t=1.55$ and 1.95. At $t=1.55$, the electron and ion flows are clearly decoupled, indicating that the reconnection is in the Hall regime. On the other hand, when the current sheet becomes elongated again at $t=1.95$, the electron and ion flows closely follow each other, indicating that Hall current does not play an important role at this time. The current sheet width in Run C only reaches a minimum of approximately $3 d_{i}$, which is why Run C never shows any onset of Hall reconnection.

\section{DISCUSSION}

An important question is, why does Run B revert to an extended current sheet after the onset of Hall reconnection? To answer this question, it is important to appreciate that although the global Lundquist number $S$ is high $\left(S=5 \times 10^{5}\right)$ for these runs, the resistivity is not negligible on the length scale of $d_{i}$. This is because $L / d_{i}$ is also a large number, which is often the case in many plasmas of interest. A relevant dimensionless parameter that quantifies how resistive the plasma is on the $d_{i}$ scale is the Lundquist number based on 


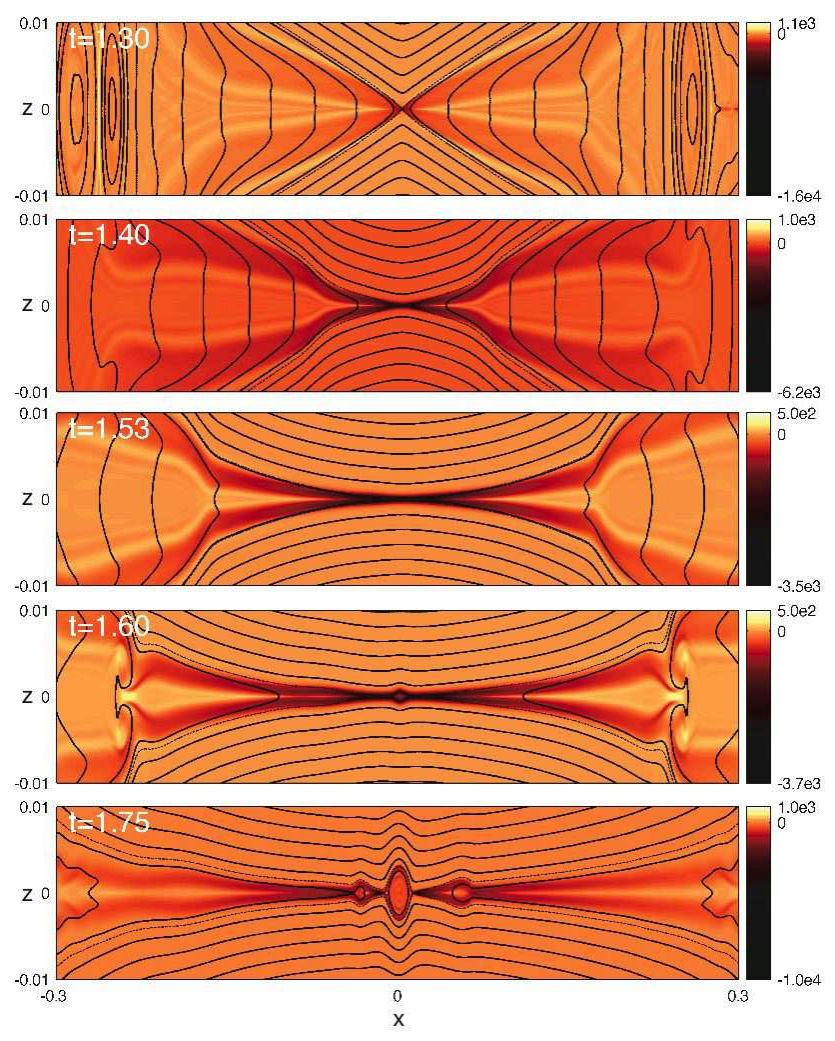

Figure 8: (Color online) Time sequence of the out-of-plane electric current density for the artificial test, overlaid with magnetic field lines. The initial condition is taken from Run A at $t=1.3$, with the ion skin depth $d_{i}$ artificially lowered from $4 \times 10^{-4}$ to $2 \times 10^{-4}$, which is the same as Run B. The opening angle between the separatrices quickly closes up, first starting from the center, then gradually propagating outward. As the current sheet becomes extended, it becomes unstable to the plasmoid instability (enhanced online).

$d_{i}$, defined as $S_{d_{i}} \equiv V_{A} d_{i} / \eta$. For Run A, B, and C, the Lundquist numbers based on $d_{i}$ are 200, 100, and 50, respectively.

Recently, it has been demonstrated by Cassak et al. that over a certain range of $S_{d_{i}}$, resistive Hall reconnection exhibits bistability, i.e. both Sweet-Parker and Hall reconnection are physically realizable. [8, 23] (Cassak et al. use the notation $\eta^{\prime}$, which is $1 / S_{d_{i}}$.) The condition for bistability may be expressed as[8]

$$
\frac{L}{d_{i}}>S_{d_{i}}>S_{d_{i}}^{c}
$$

where $S_{d_{i}}^{c}$ is a critical Lundquist number based on the $d_{i}$ scale. Here the condition $L / d_{i}>S_{d_{i}}$ is equivalent to the condition $\delta_{S P}>d_{i}$ for the existence of the Sweet-Parker solution, and 
the condition $S_{d_{i}}>S_{d_{i}}^{c}$ simply means that the plasma cannot be too resistive on the $d_{i}$ scale, otherwise the Hall solution will cease to exist. If $S_{d_{i}}>L / d_{i}$, only the Hall solution is available; and if $S_{d_{i}}<S_{d_{i}}^{c}$, only the Sweet-Parker solution is realizable. The critical value $S_{d_{i}}^{c}$ was found to be approximately 50 in Ref. [8] for a double tearing mode configuration with two Harris current sheets in a system of dimensions $409.6 d_{i} \times 204.8 d_{i}$. That study also included electron inertia, with a mass ratio of $m_{e} / m_{i}=1 / 25$.

In the present study, it appears that Run B, with $S_{d_{i}}=100$, is already below the critical value $S_{d_{i}}^{c}$ for transition, therefore the Hall solution is unstable. This implies that the critical value $S_{d_{i}}^{c}$ is greater than 100, higher than approximately 50 found by Cassak et al. To verify that the Hall solution is unstable for the set of parameters of Run B, we carry out the following test. We take the solution of Run $\mathrm{A}$ at $t=1.3$ and restart with the ion skin depth $d_{i}$ artificially lowered to $2 \times 10^{-4}$, the same value as Run B. Figure 8 shows the time sequence of this test. As a result of lowering $d_{i}$, the opening angle between the separatrices quickly closes up, first starting from the center, then gradually propagating outward. The current sheet becomes extended at the same time and eventually breaks up into plasmoids. This test confirms that the Hall solution is indeed unsustainable for the set of parameters of Run B. On the other hand, the other solution that the system will tend to make a transition to - the Sweet-Parker solution — is also unstable due to the plasmoid instability. Therefore, Run B is in a "bi-unstable" regime, and shows a continuous generation of new plasmoids.

From the above discussion, it is now clear that to realize the intermediate regime, we need the following two conditions: First, secondary current sheets must be able to reach the $d_{i}$ scale to trigger Hall reconnection. Second, the Hall solution has to be unstable, i.e. the condition $S_{d_{i}}<S_{d_{i}}^{c}$ must be satisfied. Therefore, to delineate the region of the intermediate regime in the parameter space, it is important to know how the critical value $S_{d_{i}}^{c}$ depends other dimensionless parameters. Cassak et al. [8] give an estimate of $S_{d_{i}}^{c}$ by equating the resistive diffusion across the current sheet $\eta / \delta^{2}$ with the inward convection $u_{i n} / \delta$, where $\delta$ is the current sheet width and $u_{i n}$ is the electron inflow speed. They assume that the current sheet width $\delta$ scales like the electron skin depth $d_{e}=c / \omega_{p e}$, where $\omega_{p e}$ is the electron plasma frequency, and $u_{i n}$ scales like $0.1 V_{A e}$, where $V_{A e}=B / \sqrt{4 \pi n m_{e}}$ is the electron Alfvén speed based on the magnetic field immediately upstream of the electron current layer. By using the observed upstream magnetic field $B \sim 0.3 B_{0}$, where $B_{0}$ is the asymptotic field, they obtain an estimate $S_{d_{i}}^{c} \sim 30$, which is reasonably close to the observed value $S_{d_{i}}^{c} \simeq 50$. 
The above argument might suggest that $S_{d_{i}}^{c}$ is a constant independent of system parameters, but this is open to question. For example, $S_{d_{i}}^{c}$ may depend on the ratios $L / d_{i}$ and $m_{e} / m_{i}$. This issue cannot be settled by appealing to the numerical data presented in Ref. [8], which presents results for only one set of ratios $\left(L / d_{i}=409.6\right.$ and $\left.m_{e} / m_{i}=1 / 25\right)$. In the present study, electron inertia is neglected. Therefore, we focus on the possible dependency of $S_{d_{i}}^{c}$ on $L / d_{i}$. To determine $S_{d_{i}}^{c}$ for each system size requires many runs and large computational resources, which are not practical at the present time. Instead, we perform a series of simulations with $S_{d_{i}}=100$, same as Run B, but with smaller system sizes. Our results suggest that $S_{d_{i}}^{c}$ increases with increasing system size $L / d_{i}$.

We perform two simulations with $L / d_{i}=500,1000$ and $S=5 \times 10^{4}, 10^{5}$, respectively. Also, we restart Run A at $t=1.3$, but lower the Lundquist number $S$ to $2.5 \times 10^{5}$. As such, we have three additional runs with $L / d_{i}=500,1000$, and 2500 , all with the same $S_{d_{i}}=100$. For the first two runs, the Sweet-Parker layer becomes unstable to the plasmoid instability, which quickly triggers onset of Hall reconnection. The onset of Hall reconnection expels all the plasmoids, and the reconnection precedes in the same manner as Run A. For the third run, the diffusion region broadens quickly after the Lundquist number is lowered, with the current sheet width increasing from $0.15 d_{i}$ to $0.65 d_{i}$. After that, the system remains in a quasi-steady X-point geometry. These results indicate that for these smaller system sizes, the critical value $S_{d_{i}}^{c}$ is smaller than 100 ; whereas for Run B, with $L / d_{i}=5000$, the critical value $S_{d_{i}}^{c}$ is greater than 100 .

A closer comparison of the dissipation regions for these runs reveals some interesting features. We observe a consistent trend that in the quasi-steady phase, the larger the system size is, the smaller is the opening angle in the downstream region. Furthermore, the current sheet is found to be longer and wider, when normalized to $d_{i}$, for a larger system. Figure 9 shows the separatrices of the three runs when the reconnected fluxes are approximately the same $(\simeq 0.027)$. The upper panel shows the entire simulation domain. The proximity of the three curves indicates that the global conditions are similar. However, a close-up view around the X-point, shown in the lower panel, reveals that the opening angle in the downstream region is smaller for a larger system. Likewise, in Figure 10 we plot current density profiles along the inflow and the outflow directions. Here we normalize lengths to $d_{i}$ and the current density to the peak value. Clearly, the current sheet length and width increase with increasing the system size $L / d_{i}$. Finally, these runs are all well resolved with 

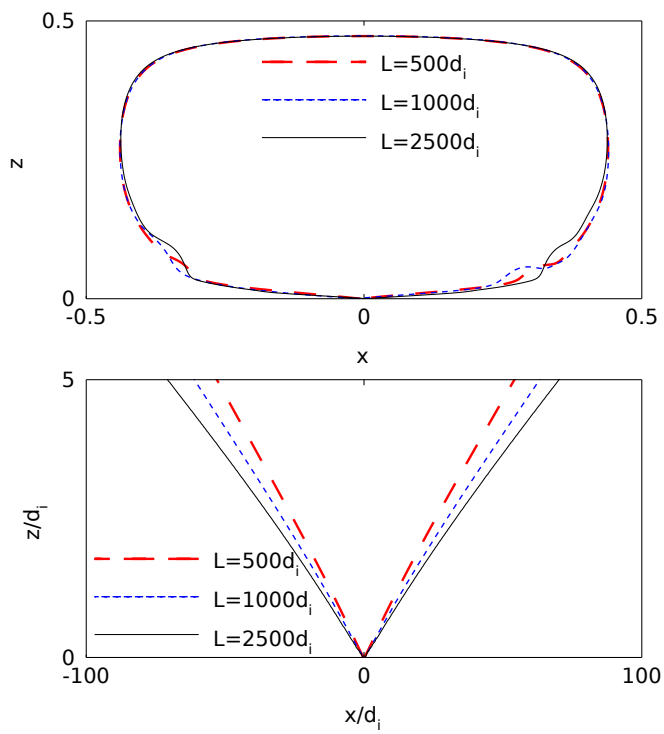

Figure 9: (Color online) The separatrices of the three runs when the reconnected fluxes are approximately the same $(\simeq 0.027)$. Only the region $z>0$ is shown. Upper panel: the whole simulation domain. Lower panel: a close-up view around the X-point. Note that in the lower panel the coordinates are normalized to $d_{i}$ and shifted horizontally to account for the slight misalignment of the X-point for each run. Also the $z$ direction is stretched for better visualization.

more than 30 grid points per $d_{i}$ along the inflow direction at the current sheet. Figure 11 shows the electron and ion inflows and the balance of $E_{y}=-\left(\mathbf{u}_{e} \times \mathbf{B}\right)_{y}+\eta J_{y}$ in the generalized Ohm's law, for the case $L / d_{i}=1000$. The $-\left(\mathbf{u}_{e} \times \mathbf{B}\right)_{y}$ term and $\eta J_{y}$ term add up to a nearly uniform out-of-plane electric field $E_{y}$, as required for quasi-steady reconnection in two dimensions. This indicates that the current sheet is well resolved and the reconnection is supported by resistivity, rather than numerical dissipation.

The fact that the current sheet width $\delta$ increases monotonically with increasing $L / d_{i}$ for the same $S_{d_{i}}$ suggests that the critical value $S_{d_{i}}^{c}$ also increases monotonically with $L / d_{i}$. This is evident from the following thought experiment. Suppose we start from a Hall solution, and gradually lower $S_{d_{i}}$ by increasing $\eta$, the current sheet width $\delta$ will gradually increase. Because the current sheet in Hall reconnection has to be thinner than $d_{i}$, the Hall solution will cease to exist when the current sheet width is approaching $O\left(d_{i}\right)$. (More precisely, the Hall solution will cease to exist when its width is equal to the width of the unstable solution[26], i.e. when the stable fixed point and the unstable fixed point annihilate each 
other; see the discussion in Ref. [23].) Since the current sheet width increases in a larger system for the same $S_{d_{i}}$, the width will approach $O\left(d_{i}\right)$ at a higher $S_{d_{i}}$ in a larger system. Consequently, a larger system has a higher critical value $S_{d_{i}}^{c}$. This conclusion is consistent with our finding that $S_{d_{i}}^{c}>100$ for $L / d_{i}=5000$ while $S_{d_{i}}^{c}<100$ for the three smaller systems.

Intuitively, the dependence of the dissipation region on the system size may be understood as a competition between the attraction of the two coalescing islands on the global scale, which tends to close up the downstream region and make the current sheet extended, and the Hall physics at the local $d_{i}$ scale, which opens up the downstream region. Our results indicate that to have a complete understanding of what determines $S_{d_{i}}^{c}$, we need a theory that couples local reconnection physics with the global configuration. Although the precise scaling is unknown at this time, the fact that $S_{d_{i}}^{c}$ increases with increasing $L / d_{i}$ has profound implications on the accessibility of the intermediate regime in large systems. Note that the line that separates region (3) and region (4) in Figure 1 has a constant $S_{d_{i}}$. Therefore, if $S_{d_{i}}^{c}$ is independent of $L / d_{i}$, conceivably the intermediate regime will be a narrow region between region (3) and region (4). Now we have shown that $S_{d_{i}}^{c}$ increases with increasing $L / d_{i}$. That implies that it will be easier to access the intermediate regime for larger systems. Therefore, it is important to determine the precise scaling of $S_{d_{i}}^{c}$ with respect to $L / d_{i}$. Such a study requires the investment of significant computational resources, and is left to the future work. Finally, when more sophisticated models are employed, the condition for transition may also depend on some other dimensionless parameters as well.

The existence of the intermediate regime and the dependence of $S_{d_{i}}^{c}$ on $L / d_{i}$ in other global configurations remain to be studied. The present island coalescence configuration differs from the more commonly studied tearing mode configuration in one important aspect. The island coalescence configuration has the attractive force between the two merging islands as an "ideal" drive of reconnection, which is absent in the tearing mode configuration. Could this be the reason that the intermediate regime has not been found yet in the standard tearing mode configuration? We do not know the answer. However, it should be noted that previous Hall MHD simulations all have substantially smaller system sizes, which, according to our findings, may make the intermediate regime less likely to occur. Only future studies with much larger system sizes could possibly answer this question.

In light of the present study, we would like to comment on the recent controversy regarding 

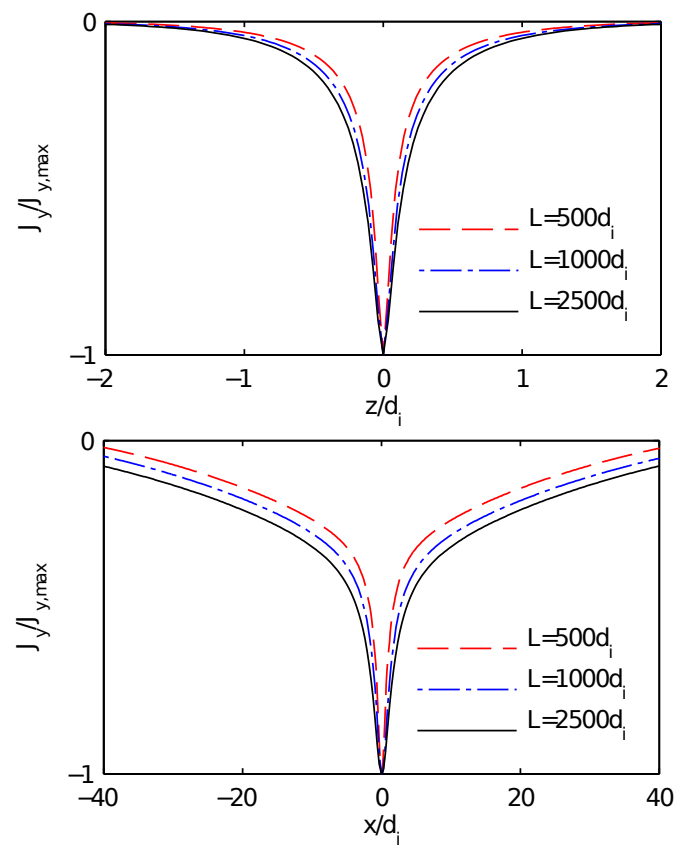

Figure 10: (Color online) Current density profiles alone the inflow (upper panel) and the outflow (lower panel) directions. Here we normalize the coordinates to $d_{i}$ and the current density to the peak value.
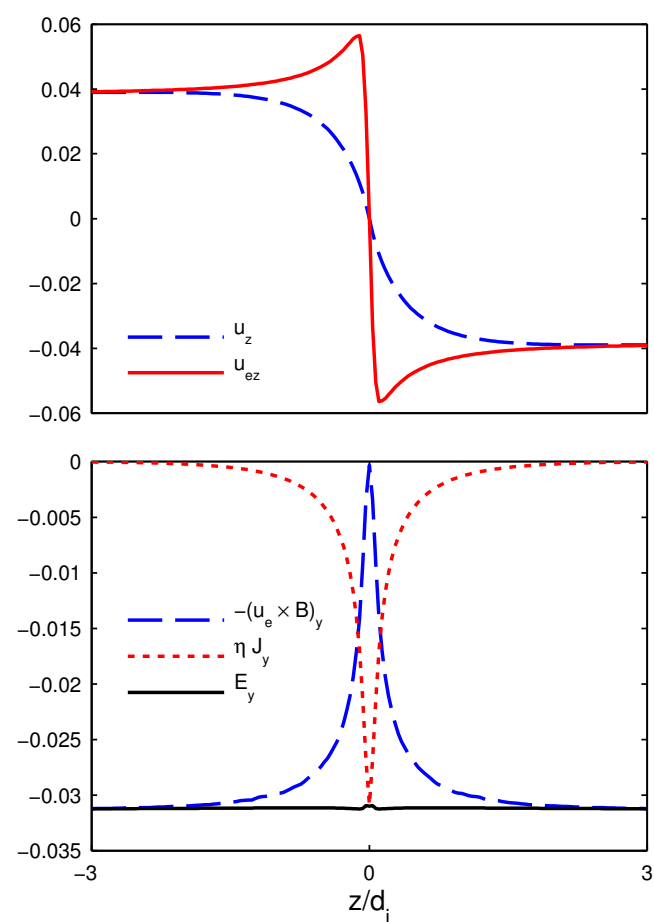

Figure 11: (Color online) The electron and ion inflows (upper panel) and the balance of $E_{y}=$ $-\left(\mathbf{u}_{e} \times \mathbf{B}\right)_{y}+\eta J_{y}$ (lower panel) in the generalized Ohm's law, for the case $L / d_{i}=1000$ 
the role of electron inertia effects on bistability. [23 [25] Whereas Zocco et al. [24] claim that electron inertia is essential, Cassak et al. [23] argue that it is Hall physics rather than electron inertia that is responsible for bistability. The conclusion of Cassak et al. is supported by an independent study by Sullivan et al. [25] Run A in the present study may be interpreted as an independent verification of the claim made by Cassak et al. [23] and Sullivan et al. [25] that bistability survives even in the absence of electron inertia. In Run A, the Hall solution is realized and remains stable after onset of the plasmoid instability. However, the SweetParker solution clearly exists, because the Sweet-Parker width $\delta_{S P} \simeq 10^{-3}$ is significantly above the ion skin depth $d_{i}=4 \times 10^{-4}$. Had it not been for the intervention of the plasmoid instability, Run A would have realized the Sweet-Parker solution. Therefore, within the framework of the original bistability theory, when the plasmoid instability is not taken into account, both Sweet-Parker and Hall solutions are realizable for the set of parameters of Run A, and the system is bistable. When the plasmoid instability is included, the SweetParker solution in Run A becomes physically unrealizable, and the Hall solution is the only possibility. The present study is valuable as an independent test of the phenomenon of bistability because it is done with a different code and a different initial condition. (Both Cassak et al. and Sullivan et al. use the code F3D.[27])

However, Cassak et al. [23] further claim that balancing the Hall term and the resistive term in the out-of-plane component of the generalized Ohm's law (as was done in Refs. [24, 28 31] ) corresponds to an unstable, and thus physically unrealizable, solution. As such, compressing the current layer leads to a runaway toward smaller scales. They argue that the runaway process "stops only when additional physics, such as off-diagonal elements of the pressure tensor, become important at electron scales," and "in two-fluid simulations of Hall reconnection, the runaway process is often stopped using an explicit high order dissipation term such as hyperviscosity or through numerical dissipation because off-diagonal pressure tensor terms are absent from the model." (See the discussion in Sec. II of Ref. [23].) This claim is inconsistent with our own study, where we have found that the $\eta J_{y}$ term by itself can balance the reconnection out-of-plane electric field around the X-point in robust Hall reconnection without the assistance of higher order dissipation terms, as shown in Figure 11. Supporting the reconnection electric field solely by resistivity has been shown before by Wang et al. [32] and recently by Sullivan et al. [25]. Along with this study, we have carefully verified this result via convergence tests on smaller systems. 
Because resistivity by itself can stop the current sheet from collapsing in a Hall solution without the need for other physics on electron scales, the assumption made in the argument by Cassak et al. [8] that the current sheet width in Hall solution scales as $d_{e}$ is debatable in a resistive plasma. For example, the current sheet in Figure 11 is significantly wider than $d_{e} \simeq d_{i} / 43$, when the real mass ratio of a hydrogen plasma is employed. An estimate of the contribution from the neglected electron inertia terms in the generalized Ohm's law indicates that those terms are much smaller, therefore the assumption of neglecting them is justified.

We emphasize that although resistivity can in principle balance the reconnection electric field in a Hall solution, such a balance need not necessarily be the case in Nature. It is clear that if the current sheet width approaches the $d_{e}$ scale, electron physics will come into play. However, in the thought experiment discussed above, the current sheet width increases as we increase $\eta$. There exists a certain range of $\eta$ where the current sheet width is above $d_{e}$ but the solution remains in the Hall branch. In this range of $\eta$ the reconnection electric field should mostly be balanced by the $\eta J$ term. This thought experiment has been carried out numerically by Cassak et al. [8] Contrary to what we have suggested, the simulations show that the current sheet widths in the Hall branch remain close to the $d_{e}$ scale when $\eta$ increases, before a sudden transition to a much broader current sheet in the Sweet-Parker branch (see Figure 3 of Ref. [8]). This may be due to the high electron mass $\left(m_{e}=m_{i} / 25\right)$ employed, and consequently the $d_{e}$ and $d_{i}$ scales are not sufficiently well separated.

In summary, we find that the bistability theory of Cassak et al. remains a very useful concept even in the presence of the plasmoid instability, and it greatly helps in interpreting our simulation results. However, our results also indicate the present understanding of what governs the extension of the current sheet and the stability of a Hall solution is still incomplete. In particular, much needs to be done regarding how global conditions may affect the local reconnection site. [33]

\section{CONCLUSION}

Our results show that the transition to fast reconnection in large, high-Lundquist-number plasmas can be realized by a complex interplay between the plasmoid instability and Hall reconnection. We have clearly demonstrated that the plasmoid instability can facilitate the 
onset of Hall reconnection, in a regime where Hall reconnection would otherwise remain inaccessible because the criterion $d_{i}>\delta_{S P}$ is not met (Runs A and B). However, the onset of Hall reconnection does not always lead to a single X-point topology, with all plasmoids expelled. Run B demonstrates the possibility that a single X-point geometry is itself unstable, and after the onset of Hall reconnection, reverts to an extended current sheet of the type that led to an X-point in the first place. In this case, the reconnection is characterized by sporadic, bursty behavior with new plasmoids constantly being generated. Because of the intermittent onset of Hall reconnection, on average the reconnection rate is higher than it is when the plasmoid instability does not trigger Hall reconnection (Run C), but lower than it is when a robust Hall reconnection site forms (Run A).

The results presented here may provide a possible starting point to resolve a dichotomy in the existing literature - the X-point geometry in Hall reconnection [5, ㅁ, 8, 17], versus the extended current sheet geometry embedded with plasmoids in fully kinetic simulations(14, 34 37]. Our results demonstrate that the dichotomy is false. We have shown that for some range of parameters (Run B) resistive Hall MHD allows the current sheet to become extended again after the onset, and subsequently new plasmoids are generated. That is not to say that the physical mechanisms that cause the extension of the current sheet is the same in the present Hall MHD simulations and fully kinetic simulations. Full kinetic simulations show extended current sheet and plasmoid formation even in the absence collisions, [34, 35, 37] which is not possible in the present simple fluid model, as lack of collisions means $\eta \rightarrow 0$ in the present model. Even when Run B is compared with collisional PIC simulations [14, 36] there are discernible differences. For example, PIC simulations show a continuous generation of new plasmoids. That is quite different from Run B, where the reconnection geometry goes to a singe $\mathrm{X}$-point configuration first then becomes extended again, which triggers plasmoid formation.

The results of this work may be tested in the next generation of Magnetic Reconnection Experiment (MRX), which is planned to systematically explore different regimes in the reconnection "phase diagram". [38] It may also be applicable to magnetic reconnection in laser produced high energy density plasmas, which have been the subject of great interest recently. [39 44] For applications in space and astrophysical systems, it is clear that if Spitzer resistivity is assumed, the intermediate regime in the present study is unlikely to be relevant in systems such as solar corona and Earth's magnetosphere, where a simple estimate gives 
$S_{d_{i}} \sim 10^{7}$ for solar corona (assuming $n \sim 10^{9} \mathrm{~cm}^{-3}, B \sim 100 \mathrm{G}, \mathrm{T} \sim 100 \mathrm{eV}$ ) and $S_{d_{i}} \sim 10^{10}$ for Earth's magnetotail (assuming $n \sim 1 \mathrm{~cm}^{-3}, B \sim 10^{-4} \mathrm{G}, T \sim 100 \mathrm{eV}$ ). It should be borne in mind, however, that the applicability of Spitzer resistivity in those systems is also open to debate, as the relevant resistivity may be due to wave particle interaction or other mechanisms. On the other hand, in solar chromosphere, due to variation in the plasma density of about seven orders of magnitude, $S_{d_{i}}$ based on Spitzer resistivity varies from $10^{-3}$ to $10^{4}$ (assuming $n \sim 10^{10}-10^{17} \mathrm{~cm}^{-3}, B \sim 100 \mathrm{G}, \mathrm{T} \sim 1 \mathrm{eV}$ ). Therefore, it is likely that there is some region in the chromosphere where the intermediate regime is directly applicable.

In conclusion, although the resistive Hall MHD model has limitations, the fact that the single X-point geometry is not inevitable in the Hall MHD model opens the possibility of realizing extended current sheets in global Hall MHD simulations of large systems. In future work, through the implementation of more sophisticated closures, e.g. for the electron pressure tensor term in the generalized Ohm's law, one may be able to parameterize some key kinetic effects in reconnection simulations. Progress along this direction may be essential in order to extend global modeling codes to include two-fluid and kinetic effects, as fully kinetic simulations of large systems, with realistic physical parameters are likely to remain computationally too expensive even in the near-future era of exascale computing.

\section{Acknowledgments}

This work is supported by the Department of Energy, Grant No. DE-FG02-07ER46372, under the auspice of the Center for Integrated Computation and Analysis of Reconnection and Turbulence (CICART), the National Science Foundation, Grant No. PHY-0215581 (PFC: Center for Magnetic Self-Organization in Laboratory and Astrophysical Plasmas), NASA Grant Nos. NNX09AJ86G and NNX10AC04G, and NSF Grant Nos. ATM0802727, ATM-090315 and AGS-0962698. YMH is partially supported by a NASA subcontract to the Smithsonian Astrophysical Observatory's Center of Astrophysics, Grant No. NNM07AA02C. Computations were performed on facilities at National Energy Research Scientific Computing Center. YMH would like to thank Dr. Naoki Bessho for commenting on an earlier version of the paper, and Prof. Paul Cassak for beneficial communications when the paper was under revision. We are grateful for the comments of the anonymous 
reviewers, which have helped improve this work.

[1] E. G. Zweibel and M. Yamada, Annu. Rev. Astron. Astrophys. 47, 291 (2009).

[2] A. Bhattacharjee, Z. W. Ma, and X. Wang, Phys. Plasmas 8, 1829-1839 (2001).

[3] P. A. Sweet, Nuovo Cimento Suppl. Ser. X 8, 188 (1958).

[4] E. N. Parker, Astrophys. J. Suppl. 8, 177-211 (1963).

[5] Z. W. Ma and A. Bhattacharjee, Geophys. Res. Lett. 23, 1673-1676 (1996).

[6] J. C. Dorelli and J. Birn, Geophys. Res. 108, 1133 (2003).

[7] A. Bhattacharjee, Annu. Rev. Astron. Astrophys. 42 365-384 (2004).

[8] P. A. Cassak, M. A. Shay, and J. F. Drake, PRL 95, 235002 (2005).

[9] M. Yamada, Y. Ren, H. Ji, J. Breslau, S. Gerhardt, R. Kulsrud, and A. Kuritsyn, Phys. Plasmas 13, 052119 (2006).

[10] N. F. Loureiro, A. A. Schekochihin, and S. C. Cowley, Phys. Plasmas 14, 100703 (2007).

[11] B. Coppi, E. Galvao, R. Pellat, M. N. Rosenbluth, and P. H. Rutherford, Sov. J. Plasma Phys. 2, 533 (1976).

[12] A. Bhattacharjee, Y.-M. Huang, H. Yang, and B. Rogers, Phys. Plasmas 16, 112102 (2009).

[13] Y.-M. Huang and A. Bhattacharjee, Phys. Plasmas 17, 062104 (2010).

[14] W. Daughton, V. Roytershteyn, B. J. Albright, H. Karimabadi, L. Yin, and K. J. Bowers, PRL 103, 065004 (2009).

[15] P. A. Cassak, M. A. Shay, and J. F. Drake, Phys. Plasmas 16, 120702 (2009).

[16] K. Shibata and S. Tanuma, Earth Planets Space 53, 473 (2001).

[17] L. S. Shepherd and P. A. Cassak, PRL 105, 015004 (2010).

[18] S. I. Braginskii, Rev. Plasma Physics 1, 205 (1965).

[19] H. Dreicer, Phys. Rev. 115, 238 (1959).

[20] P. N. Guzdar, J. F. Drake, D. McCarthy, A. B. Hassam, and C. S. Liu, Phys. Fluids B 5, 3712 (1993).

[21] S. Gottlieb, C.-W. Shu, and E. Tadmor, SIAM Rev. 43, 89 (2001).

[22] R. J. Spiteri and S. J. Ruuth, SIAM J. Numer. Anal. 40, 469 (2002).

[23] P. A. Cassak, M. A. Shay, and J. F. Drake, Phys. Plasmas 17, 062105 (2010).

[24] A. Zocco, L. Chacón, and A. N. Simakov, Phys. Plasmas 16, 110703 (2009). 
[25] B. P. Sullivan, A. Bhattacharjee, and Y.-M. Huang, Phys. Plasmas 17, 114507 (2010).

[26] P. A. Cassak. J. F. Drake, M. A. Shay, and B. Eckhardt, PRL 98, 215001 (2007).

[27] M. A. Shay, J. F. Drake, M. Swisdak, and B. N. Rogers, Phys. Plasmas, 11, 2199 (2004).

[28] L. Chacón, A. N. Simakov, and A. Zocco, PRL 99, 235001 (2007).

[29] A. N. Simakov and L. Chacón, PRL 101, 105003 (2008).

[30] L. M. Malyshkin, PRL 101, 225001 (2008).

[31] L. M. Malyshkin, PRL 103, 235004 (2009).

[32] X. Wang, A. Bhattacharjee, and Z. W. Ma, PRL 87, 265003 (2001).

[33] A. Kuritsyn, H. Ji, S. P. Gerhardt, and M. Yamada, Geophys. Res. Lett., 34, L16106 (2007).

[34] J. F. Drake, M. Swisdak, H. Che, and M. A. Shay, Nature 443, 553 (2006).

[35] W. Daughton, J. Scudder, and H. Karimabadi, Phys. Plasmas 13, 072101 (2006).

[36] W. Daughton, V. Roytershteyn, B. J. Albright, H. Karimabadi, L. Yin, and K. J. Bowers, Phys. Plasmas 16, 072117 (2009).

[37] A. Klimas, M. Hesse, S. Zenitani, and M. Kuznetsova, Phys. Plasmas 17, 112904 (2010).

[38] H. Ji, M. Yamada, S. Prager, W. Daughton, and V. Roytershteyn, "A Next Generation Magnetic Reconnection Experiment: Accessing "Reconnection Phase Diagrams" for Space and Astrophysical Relevances", Bull. Am. Phys. Soc., 55, 15, NP9 82 (2010).

[39] P. M. Nilson, et al., PRL 97, 255001 (2006).

[40] C. K. Li, et al., PRL 99, 055001 (2007).

[41] P. M. Nilson, et al., Phys. Plasmas 15, 092701 (2008).

[42] L. Willingale, et al., Phys. Plasmas 17, 043104 (2010).

[43] J. Zhong, et al., Nature Physics 6, 984 (2010).

[44] W. Fox, A. Bhattacharjee, and K. Germaschewski, PRL 106, 215003 (2011). 